\title{
Predictive road safety impact assessment of traffic management policies and measures
}

\author{
Ioannis Kaparias \\ Transportation Research Group \\ University of Southampton \\ Boldrewood Innovation Campus \\ Burgess Road, Southampton SO16 7QF, UK \\ Email: i.kaparias@southampton.ac.uk
}

\author{
Peng Liu \\ School of Mathematics, Computer Science \& Engineering \\ City, University of London \\ Northampton Square, London EC1V 0HB, UK
}

Antonios Tsakarestos

Chair of Traffic Engineering and Control

Technische Universität München

Arcisstraße 21, 80333 München, Germany

Niv Eden

Transportation Research Institute

Technion - Israel Institute of Technology

Technion City, Haifa 32000, Israel

\section{Pierre Schmitz}

Bruxelles Mobilité

Brussels Capital Region

Rue du Progrés 80, Brussels 1035, Belgium

\section{Suzanne Hoadley}

POLIS - European Cities and Regions Networking for Innovative Transport Solutions Rue du Trône 98, Brussels 1050, Belgium

\section{Susanna Hauptmann}

International Traffic \& Transport Associations

Kapsch TrafficCom

Am Europlatz 2, Vienna 1120, Austria 


\title{
Predictive road safety impact assessment of traffic management policies and measures
}

\begin{abstract}
In recent research the CONDUITS performance evaluation framework for traffic management and Intelligent Transport Systems (ITS) was developed, consisting of a set of Key Performance Indicators (KPIs) for the strategic themes of traffic efficiency, safety, pollution reduction and social inclusion. Follow-up work has concentrated on integrating the developed CONDUITS KPIs with microscopic traffic simulation. The outcome has been a predictive evaluation tool for traffic management and ITS, called CONDUITS_DST, in which two of the four KPI categories have been integrated previously: pollution and traffic efficiency. The objective of the present study is to further extend the predictive evaluation framework to include the theme of traffic safety. Contributing to the development of the CONDUITS_DST traffic safety module, the paper identifies and proposes relevant models and metrics linking traffic characteristics with road safety impacts. In doing so, it enables the extraction of the necessary input data for each of the three CONDUITS KPIs for traffic safety (accidents, direct impacts, and indirect impacts) directly from microscopic traffic simulation models. The proposed models and metrics are tested in conjunction with the relevant CONDUITS KPIs for safety using data from simulation models before and after the implementation of a bus priority signalling system in Brussels. Testing takes place both at the network level, but also at the level of individual links, and the results show that the framework is able to capture the expected safety impacts adequately well, paving the way towards its implementation is the traffic safety module of CONDUITS_DST.
\end{abstract}

\section{INTRODUCTION}

Cities today share common transport problems and objectives with respect to road traffic management, and put great focus on Intelligent Transport Systems (ITS). The market offers decision-makers a variety of solutions, from which they are required to choose the most suitable and effective ones. Making this choice is a non-trivial task, however, especially given that transport problems are multi-dimensional by nature. In particular, the political decision-making level needs a way to easily and quickly understand trends, appraise approximate outcomes and identify best practices in ITS and traffic management that does not require having expert knowledge in transport engineering. Hence, a performance evaluation framework that addresses the various dimensions of transport problems, while at the same time reflecting the perspectives and priorities of decision-makers, is required (Zavitsas et al., 2011).

In recent research work (FP7 CONDUITS) such an evaluation framework was formulated, consisting of a set of Key Performance Indicators (KPIs) for four strategic themes of road traffic management: efficiency, safety, pollution reduction and social inclusion (Kaparias et al., 2011). The themes were chosen in accordance with relevant policy fields, rather than areas of expert interest, so that they can address the political level of transport authorities and administrations. The main aim was to bridge the gap between experts, usually found in specialised departments of administrations, and political decisionmakers and procurers, who usually have only limited knowledge of the technical details of the transport field. The KPIs were subsequently validated through before- and after- evaluation of real-world case studies in the cities of Paris, Rome, Tel Aviv and Munich, using real data supplied by the corresponding local authorities and transport operators (Tsakarestos et al., 2011; Kaparias et al., 2012a). Through the conduct of the case studies, it was concluded that the KPIs were easy to apply and required already available data, thus forming a very useful evaluation tool for assisting decision-makers in the field of road traffic management and ITS, and to some extent for identifying best practice and lessons learnt elsewhere.

Yet the necessity for extending the CONDUITS framework from its current state of a tool for evaluating existing systems to a tool for evaluating future systems becomes apparent, given the current economic 
climate and the increasing need of making as informed decisions as possible. Follow-up work within the context of the CONDUITS-DST spinoff project, sponsored by Kapsch TrafficCom, has concentrated on integrating the CONDUITS KPIs with microscopic traffic simulation. The outcome has been a predictive evaluation tool for road traffic management and ITS, called CONDUITS_DST, in which two of the four KPI categories have been integrated to date: the pollution generated by the various transport modes in the form of emissions of both global and local pollutants, and the traffic efficiency, expressed through measures such as travel time and network reliability. Preliminary testing of the tool in three European cities (Brussels, Stuttgart and Tel Aviv) using existing microscopic traffic simulation models has, again, confirmed the validity of the methodology and has demonstrated the viability, usefulness and timeliness of the approach (Eden et al., 2012; Eden et al., 2013; Kaparias et al., 2016).

The main advantages of the predictive evaluation approach using the KPIs and CONDUITS_DST are that it provides the means to present results to non-expert audiences (such as politicians) in a simple, fast and effective way, but also that it allows estimating impacts of policies and measures before they actually occur. And while for the evaluation in terms of traffic efficiency and pollution the latter is a useful feature that contributes to the effectiveness of the transport policy- and decision-making process, for the theme of traffic safety it is absolutely critical, as conclusions need to be reached and decisions be taken before any accidents happen.

In current practice the evaluation of safety is carried out either by interpreting accident statistics for long-term decisions, such the planning of infrastructure, or by using real time surrogate safety measures for supporting control decisions in ITS and intelligent vehicles. Therefore, a prediction horizon is not available at all in the former case (as accident statistics only become available post-implementation), while in the latter case the horizon is focused on the mere short-term. The contribution of CONDUITS_DST lies in the ability to provide a priori estimates of traffic safety impacts using surrogate measures relating to traffic characteristics, as available from microscopic traffic simulation, in order to influence long-term decisions; historical data (e.g. accident numbers), then, when available, would only be used as a complement. This is a similar approach, in principle, to the telematics-enabled methods used by insurance companies to provide usage-based vehicle insurance products (e.g. Zhao, 2002; Handel at al., 2014; US National Association of Insurance Commissioners, 2019).

The objective of the present paper is to further extend the predictive evaluation framework to include the theme of traffic safety, with a view of implementing the outcome as a new module in CONDUITS_DST in future. Towards that objective, relevant models and metrics linking traffic characteristics with road safety impacts are identified and proposed, which enable the extraction of the necessary input data for each of the three CONDUITS KPIs for traffic safety (accidents, direct impacts, and indirect impacts) directly from microscopic traffic simulation models. The proposed models and metrics are then tested in conjunction with the KPIs using data from microscopic traffic simulation models before and after the implementation of a bus priority signalling system in the city of Brussels.

The paper is structured as follows: Section 2 introduces the background of the study, including the CONDUITS performance evaluation framework (KPIs) for traffic safety and a review of existing traffic safety impact assessment measures from the literature. Section 3 then goes on to formulate the methodology for the predictive safety evaluation in terms of accident numbers, direct safety impacts and indirect safety impacts. The results of the testing of the method on the case study in Brussels are reported in Section 4, along with a discussion of the analysis carried out. Finally, Section 5 concludes the paper and identifies areas of future work.

\section{BACKGROUND}

\subsection{The CONDUITS traffic safety KPIS}

Performance measures have the ability to effectively evaluate the outputs of specific solutions. However, when attempting to conduct a higher-level evaluation through a multi-dimensional benchmarking 
scheme comparing different cities with each other, performance measures are generally not suitable. The reason is that such a task necessitates the systematic and synthetic description of the cities' transport policies and infrastructures and the analysis of their impacts, which can only be expressed by a set of measures reflecting each individual scheme evaluated (Cianfano et al., 2011). This issue creates difficulties in the communication of the results to non-technical audiences, such as politicians and the general public, and a common way to deal with it is to combine individual performance measures into composite performance indices (KPIs) (Cambridge Systematics Inc, 2005; Zhou et al., 2006).

The main advantage of KPIs is simplicity, as it is much easier to understand and grasp a single number rather than a large collection of individual measures, whose meaning often requires trained insight and careful analysis. The disadvantage, nevertheless, is that an aggregate number does not provide immediate insight into which aspects of the performance are changing or why, making it difficult to distinguish the sensitivity of an index to changes in its component measures. However, this ambiguity may lead to some other advantages. The index increases the opportunity for all modes and markets to be included, conveys the idea that each service is important, and elevates the discussion about how to best measure and report system performance. This cooperation between modes and sectors enhances awareness, broadens perspectives and leads to more comprehensive solutions.

In line with the European Commission's strategy on the future of transport, as presented in the 2001 and 2011 white papers (European Commission, 2001; 2011), a performance evaluation framework was defined by the FP7 CONDUITS project, consisting of a set of measures and KPIs for the four strategic themes of traffic efficiency, traffic safety, pollution reduction, and social inclusion (Kaparias et al., 2011). Among the KPIs of the complete framework, this study focuses on the theme of traffic safety, which is addressed through three indices, namely accidents, direct safety impacts and indirect safety impacts.

Starting from the accidents index, this relies on the quantification of the safety impact as the number of people injured or killed (Elvik et al., 2009), and hence the main factors considered in the KPI are the ones influencing road injuries: exposure (the amount of travel), accident rate (the risk of accident per unit of exposure), and accident severity (the outcome of accidents concerning injuries). The accidents KPI is thus formulated as follows:

$$
I_{A C D}=\sum_{l}\left\{w_{l} \cdot \sum_{s e}\left[w_{s e} \cdot \sum_{m}\left(w_{m} \cdot \frac{A C D_{l, s e, m}}{D T V_{l}}\right)\right]\right\}
$$

where $I_{A C D}$ is the value of the accidents KPI (with lower values indicating lower accident impacts, and hence better performance), $w_{\text {se }}$ denotes the weighting factor representing the importance of reducing the number of casualties in accidents with a specific severity se from the set of possible severity levels (uninjured, slightly injured, seriously injured or killed), $w_{m}$ is the weighting factor representing the importance of reducing the number of casualties in accidents involving a specific traffic mode $m$ from the set of possible traffic modes (car, truck, bus, motorcycle, bicycle, pedestrian, etc.), $w_{l}$ is the weighting factor representing the importance of link (or junction) $l$ among the set of links (and junctions) of the network in terms of safety, $A C D_{l, s e, m}$ is the number of casualties of severity se involving users of mode $m$ on link $l$ on an average day, and $D T V_{l}$ is the daily traffic volume on link $l$ in million vehicles.

It should be noted here that the use of the accident rate measure, which is based here on the Daily Traffic Volume (DTV), is in order to normalise the raw number of accidents. This instance does not imply a correlation between traffic volume and the number or severity of accidents. It is rather a way to make KPI values comparable between either different network parts with different levels of traffic or between cities of different sizes. A direct comparison of absolute numbers would bias the consideration in favour of the entity with lower traffic loads. Also, the choice of "million vehicles per day" as the unit of the DTV is based on the fact that the CONDUITS KPIs are primarily intended for large scale evaluations of entire networks or large network segments, which makes the numerical value of the KPI more 
controllable.

Moving onto the direct safety impacts index, this quantifies the safety impact as the number of actions/interventions taken by systems or users, which have the objective of averting a safety-critical situation; these include, for example, vehicle braking occurrences by drivers, or collision warning events by vehicle-based systems. The direct impacts KPI is thus formulated as follows:

$$
I_{D S}=\sum_{l}\left[w_{l} \cdot \sum_{m}\left(w_{m} \cdot \frac{I N T E R V_{l, m}}{D T V_{l}}\right)\right]
$$

where $I_{D S}$ is the value of the direct safety impacts KPI (with lower values indicating lower safety impact, and hence better performance), INTER $V_{l, m}$ denotes the number of actions/interventions for mode $m$ on link (or junction) $l$ on an average day, while $w_{l}$ and $w_{m}$ are the weighting factors representing the importance of mode $m$ and link $l$ in the network, and $D T V_{l}$ is the daily traffic volume on link $l$ in million vehicles. In contrast to the accidents KPI, the direct safety impacts are linked to individual actions of a system or by road users that could lead to a critical event (i.e. accident) and not to the event itself; the focus here is on the risk of occurrence of an event, which may well not materialise in reality, despite the occurrence of critical actions.

Finally, the indirect safety impacts index considers the total duration of critical occurrences/situations, which are not necessarily a result of users avoiding a safety hazard, but which can be associated with an adverse safety impact as a side-effect. These include, for example, exceedance of the speed limit, or instances of traffic flow breakdown on motorways. The indirect safety KPI is thus formulated as follows:

$$
I_{I S}=\sum_{l}\left[w_{l} \cdot \sum_{m}\left(w_{m} \cdot \frac{C S_{l, m}}{T}\right)\right]
$$

where $I_{I S}$ is the value of the indirect safety impacts KPI (again with lower values indicating lower safety impact, and hence better performance), $C S_{l}$ denotes the total duration of critical occurrences/situations on link $l, w_{l}$ and $w_{m}$ are the weighting factors representing the importance of mode $m$ and link $l$, and $T$ is the total time of observation.

Depending on the type of evaluation, the data sources of the $A C D, I N T E R V$ and $C S$ input quantities to the KPIs vary. Specifically, in a before- and after- evaluation of an already realised/implemented scheme, the values can be obtained from actual data collected from the field. In the case of predictive evaluation of a proposed scheme, on the other hand, the values can be calculated from the output of microscopic traffic simulation models (such as PTV VISSIM, PARAMICS or AIMSUN). Naturally, this only relates to the INTERV and $C S$ quantities, as $A C D$ would not be available before implementation of the scheme. The values of the weights $w_{l}, w_{s e}$ and $w_{m}$ are the policy-aware element of the KPIs, and can be set by the decision-maker to reflect high-level policy objectives, just like it is done in the case of the pollution KPI (Kaparias et al., 2016).

\subsection{Brief overview of traffic safety impact assessment measures}

A range of safety impact assessment measures have been developed, with the primary objective of assessing the road safety condition and helping to define appropriate transport management solutions to current problems. However, these measures can also be used for safety performance assessment of implemented transport applications.

The European Transport Safety Council (2001) defines transport safety performance measures as "any measurement that is causally related to crashes or injuries, used in addition to a count of crashes or injuries in order to indicate safety performance or understand the process that leads to accidents". 
However, using the counts of accidents or injuries is usually insufficient to represent transport safety as a whole, due to a number of limitations. For example, the number of crashes or injuries depends on probability fluctuations of random events, and this implies that the underlying, long-term problems cannot necessarily be revealed by short-term recordings. Also, hazardous situations that did not cause an accident are often not recorded as near-miss incidents (Sigfridsson et al., 2001). Instead, the use of wider safety performance measures can indicate risky problems at an early stage (before accidents happen) and allows to distinguish systematic improvements form random fluctuations.

In most countries, road user behaviour and road and vehicle engineering characteristics are employed to describe road safety performance. With respect of road user behaviour, commonly used safety performance measures include (European Transport Safety Council, 2001):

- Speeding, with respect both to mean speed, variance, and speed limit violations;

- Percentage use of seat belts and child restraints;

- Percentage use of crash helmets;

- Incidence of drinking and driving;

- Failure to stop or yield at junctions or at pedestrian crossings;

- Inadequate headways - close following;

- Use of daytime running lights;

- Use of reflective devices, especially for cyclists and pedestrians; and

- Use of pedestrian crossing facilities (by pedestrians).

Many of these safety performance measures have formed the basis of various predictive safety models, which have been developed and tested both in simulation and real-world environments. For example, speed is one of the key parameters necessary to calculate time-to-collision, a fundamental variable in traffic conflicts models, such as the Swedish Traffic Conflicts Technique (Hydén, 1987). Speed is also the basis of Nilsson's power model (Nilsson, 2004), which predicts changes in accident rates as a function of average speed changes. Alternatively, car-following behaviour with shorter headways than what is considered safe is commonly viewed as a near-accident situation and hence a safety risk, and forms the basis of various models.

Most existing applications of such models focus predominantly on a particular phenomenon or localised case study rather than on a comprehensive assessment of performance of the network or parts thereof in terms of safety. Still, many such measures can be used in conjunction with the CONUITS safety KPIs, as they can provide the input necessary for their computation. This is tackled in the next section.

\section{PREDICTIVE TRAFFIC SAFETY EVALUATION METHODOLOGY}

\subsection{Methodology requirements and overview}

The objective of the predictive traffic safety evaluation methodology proposed is to obtain the necessary data for input to the CONDUITS safety KPIs, which originate from microscopic traffic simulation models. Microscopic traffic simulation is an efficient tool offering a detailed reflection of the implementation of a road traffic management or ITS measure of the mobility patterns in the transport network. The output of such a model is a so-called vehicle record file, which contains detailed information about the state of each vehicle in the network for each time point of the simulation period. Such information contains a number of attributes, including location coordinates, road geometrical features (e.g. gradient), vehicle speed, vehicle acceleration, vehicle type and information on the surrounding traffic conditions. This can be subsequently segregated and aggregated with other mobilityrelated data on different aspects of the network (nodes/links/routes) and different transport modes.

The data contained in vehicle record files can be readily input in the CONDUITS KPIs for traffic efficiency. Coupled with an appropriate emissions modelling tool (such as COPERT or AIRE II), 
appropriate input data for the KPIs for pollution can also be derived. In the case of safety, though, the required input data are not readily available, and need to be either processed, or coupled with another existing predictive safety model. The next sub-sections describe how this is done for the three KPIs introduced in Section 2.1.

\subsection{Evaluation of accident numbers}

As accident numbers are not output from microscopic traffic simulation models, it is necessary to identify a proxy, which can be used as input to the relevant KPI in equation (1). The selected measure here is the average speed. In fact, it is recognised that driving speed plays an important role in road traffic safety measurement, as higher driving speed provides less time to respond to emergencies for both drivers and pedestrians, and as high speed injects additional momentum, increasing the severity of accidents (Organisation for Economic Cooperation and Development, 2006). It has been reported that excess and unsuitable speeds are responsible for a high proportion of road accidents and contribute to about one third of fatal crashes and $12 \%$ of all road crashes as main crash causal factors (Bowie and Waltz, 1994).

Looking in more detail at the relationship between speed and accidents, various empirical studies have suggested that that for every increase of average speed by $1 \mathrm{~km} / \mathrm{h}$, the number of accidents is likely to increase 3-4\%, with more significant impacts found for serious accidents. Conversely, every $1 \mathrm{~km} / \mathrm{h}$ of reduction in the average driving speed leads to a $5 \%$ decrease of the number of fatal accidents (Bowie and Waltz, 1994; European Transport Safety Council, 2001). A more formal expression of these findings is proposed by Nilsson's power model (Nilsson, 2004), which relates average speed changes with the number of crashes by severity (Table 1).

\begin{tabular}{|c|c|}
\hline \multicolumn{2}{|c|}{ Speed measure for accidents and injuries prediction } \\
\hline Accidents (y) & Injured $(\mathrm{z})$ \\
\hline Fatal accidents & Fatalities \\
\hline $\mathrm{y}_{1}=\left(\frac{\mathrm{v}_{1}}{\mathrm{v}_{0}}\right)^{4} \mathrm{y}_{0}$ & $\mathrm{z}_{1}=\left(\frac{\mathrm{v}_{1}}{\mathrm{v}_{0}}\right)^{4} \mathrm{y}_{0}+\left(\frac{\mathrm{v}_{1}}{\mathrm{v}_{0}}\right)^{8}\left(\mathrm{z}_{0}-\mathrm{y}_{0}\right)$ \\
\hline Fatal and serious injury accidents & Fatalities and severe injuries \\
\hline $\mathrm{y}_{1}=\left(\frac{\mathrm{v}_{1}}{\mathrm{v}_{0}}\right)^{3} \mathrm{y}_{0}$ & $\mathrm{z}_{1}=\left(\frac{\mathrm{v}_{1}}{\mathrm{v}_{0}}\right)^{3} \mathrm{y}_{0}+\left(\frac{\mathrm{v}_{1}}{\mathrm{v}_{0}}\right)^{6}\left(\mathrm{z}_{0}-\mathrm{y}_{0}\right)$ \\
\hline All injury accidents & All injuries (including fatalities) \\
\hline $\mathrm{y}_{1}=\left(\frac{\mathrm{v}_{1}}{\mathrm{v}_{0}}\right)^{2} \mathrm{y}_{0}$ & $\mathrm{z}_{1}=\left(\frac{\mathrm{v}_{1}}{\mathrm{v}_{0}}\right)^{2} \mathrm{y}_{0}+\left(\frac{\mathrm{v}_{1}}{\mathrm{v}_{0}}\right)^{4}\left(\mathrm{z}_{0}-\mathrm{y}_{0}\right)$ \\
\hline
\end{tabular}

Table 1: Nilsson's power model relating accidents with speed changes

Nilsson's power model (Table 1) is a suitable method for generating input for the CONDUITS accidents KPI on the basis of microscopic traffic simulation, as it relates accident numbers with traffic characteristics. Furthermore, it has the additional advantage of flexibility, as it defines separate models based on both accident numbers (left column of Table 1) and on injury/fatality numbers (right column of Table 1), which are to be used interchangeably depending on the format of the available data.

However, Nilsson's power model relies on the fundamental assumption that reliable initial accident numbers, differentiated by type, are known, which is, however, rarely the case, as such data are often either not available at all, or statistically insignificant. As such, it is very likely that the KPI of equation (1) cannot be computed as an absolute value. Instead, the present study proposes calculating the CONDUITS KPI for accidents in relative terms, i.e. as

$$
\Delta I_{A C D}=\frac{I_{A C D, 0}-I_{A C D, 1}}{I_{A C D, 0}}
$$


where $\Delta I_{A C D}$ is the improvement (positive) or deterioration (negative) in terms of accidents, and $I_{A C D, 0}$ and $I_{A C D, 1}$ are the values of the accidents KPI before and after the change in average speed respectively. It should be noted that although the $y_{0}$ term is not known, it cancels out in equation (4), and therefore the calculation of $\Delta I_{A C D}$ is possible.

\subsection{Evaluation of direct safety impacts}

Direct safety impacts include actions/interventions taken by systems or users, which have the objective of averting a safety-critical situation, and the selected measure in this category is vehicle deceleration, which corresponds to vehicle braking occurrences. In fact, acceleration and deceleration are important properties of vehicle performance and usually lead to accidents when vehicles start and brake suddenly without sufficient safety distance (Bared at al., 1999), and hence significant changes in acceleration and deceleration are empirically considered as responses to emergency situations and indicators for rear-end collisions. For instance, studies have suggested that deceleration, which usually refers to the braking rate, is closely related to the severity of accidents (Hydén, 1996).

Using the categorisation of Hydén (1996), shown in Figure 1, the deceleration allocated in the "uncontrolled" braking zone represents a high risk level of accidents, while the "medium/hard" zone implies a less severe accident risk. "Normal" braking behaviour can be regarded as non-accident-causing in terms of deceleration. The number of occurrences of uncontrolled braking (more than $7 \mathrm{~m} / \mathrm{s}^{2}$ ), or "abnormal" (more than $2 \mathrm{~m} / \mathrm{s}^{2}$ ) is available from microscopic traffic simulation and can, hence, be used as input in the direct safety impacts KPI of equation (2). However, it should be noted that the braking behaviour is also heavily influenced by the vehicle characteristics, and hence the calculation should be differentiated by mode (car, bus, HGV, etc.) and then aggregated with appropriate weighting factors in the KPI if desirable.

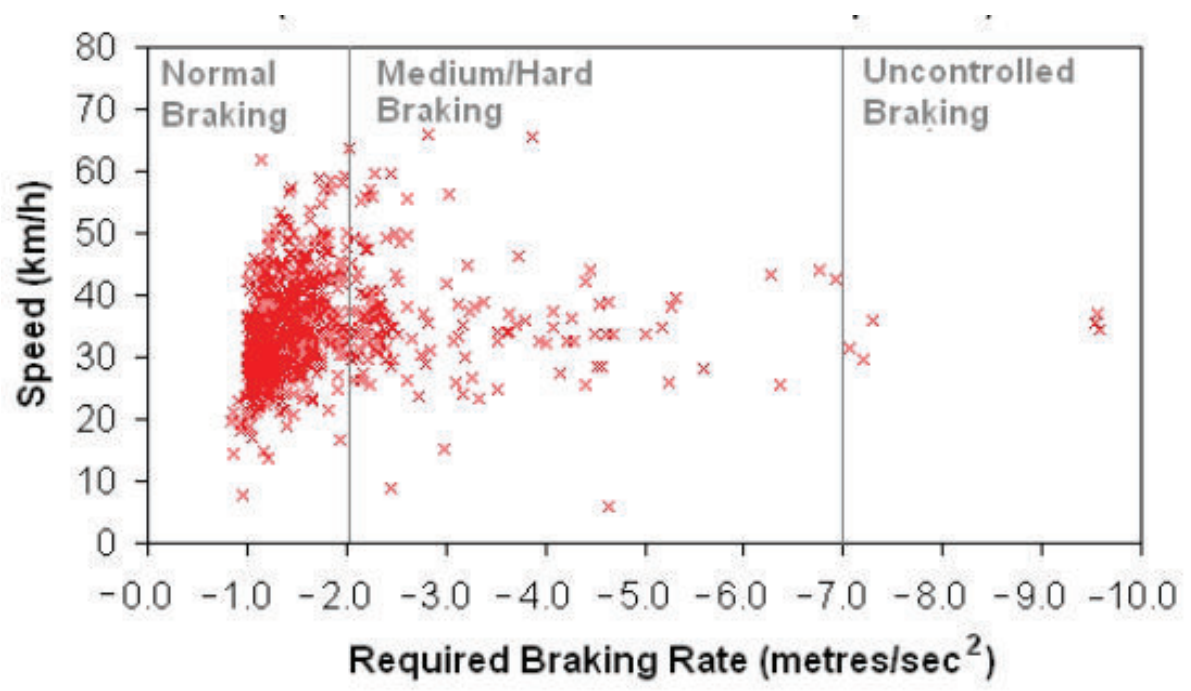

Figure 1: Severity of accident risk based on braking rate (deceleration) (Hydén, 1996)

An aspect worth mentioning here is that the KPI considers all braking events as having a negative safety impact and does not distinguish between their causes. Indeed, it can be argued that some of the braking occurrences may have causes that do not, at least in principle, entail a significant safety risk (such as traffic lights and road closures). However, the braking events are themselves associated with a certain level of accident risk, and it is therefore appropriate to consider them all without any exceptions in the KPI, in particular given that traffic management policies usually make changes to what road users are used to (e.g. changing the traffic signals to something different) and may, hence, have unintended negative safety impacts.

\subsection{Evaluation of indirect safety impacts}


Indirect safety impacts consider the total duration of critical occurrences/situations over the total duration of the period of observation. The situations considered are the ones which are not necessarily a result of users avoiding a safety hazard, but which can be associated with an adverse safety impact as a side-effect. The selected measure in this category is the speeding rate, i.e. the frequency of exceedance of the speed limit. Speeding behaviour is, in fact, one of the most commonly used indicators illustrating the relationship between driving behaviour and road safety, but it does not involve an action aimed at averting a safety hazard, so it is an appropriate measure for this category of safety impact.

The speeding rate can be extracted directly from the vehicle record files of microscopic traffic simulation packages, as the total time of exceedance of the speed limit (usually $50 \mathrm{~km} / \mathrm{h}$ in urban areas) in vehiclehours over the total time of observation, also in vehicle hours. This provides the values of the necessary $C S$ and $T$ input variables of the indirect safety impacts KPI of equation (3). The calculation can also be performed in a stratified manner disaggregated by mode, and the result can then be aggregated using appropriate weights in the KPI.

The use of the speed limit exceedances in the KPI for indirect safety impacts helps to evaluate not only the existence of a negative impact (in terms of the number of occurrences), but also its severity, as expressed by the interconnection of further related critical actions caused by the same occurrence. For example, a careless driver who is speeding will increase the value of the indirect safety impacts KPI, as expected; however, his/her behaviour may well additionally cause a collision or near-miss, which will be reflected in other vehicles braking, and will hence additionally increase the value of the direct safety impacts KPI. In contrast, a different speeding driver may not cause such a severe chain of braking occurrences; this would mean that the overall impact in that case would be lower, as there would be a similar indirect impact but a lower direct one. The consideration of chains of events/occurrences as separate instances is, therefore, important and does not constitute double-counting.

\section{APPLICATION AND RESULTS}

\subsection{Application case study}

The research described has been carried out in close cooperation with city authorities, with the predictive evaluation framework being tested through an existing case study in the city of Brussels. The BrusselsCapital Region has an extensive network of roads and public transport, with the latter comprising buses, trams and the Brussels Metro. The surface public transport network, largely operated by the Brussels Intercommunal Transport Company (STIB-MIVB), consists of $140 \mathrm{~km}$ of tram lines and $440 \mathrm{~km}$ of bus routes, serving a total of some 2200 stops across the entire region. Tram vehicles in operation include the new generation T3000 and T4000 series, which are gradually replacing older vehicles, while the bus network has a diverse fleet consisting of electric hybrid buses and some fully electric "Citybuses" alongside the older conventional buses that are in the process of being phased out (STIB-MIVB, 2019).

The most important condition for the suitability of the case study has been for it to be based on an already concluded planning scheme, such that a calibrated simulation model that has been already used in the planning process could be made available. In this way, the CONDUITS_DST study could then concentrate on the calculation and analysis of the impacts rather than the further development and calibration of the simulation model.

The case study provided by the Brussels-Capital Region has been part of a wider programme aiming at increasing the operational speed of most of its public transport lines. The programme focuses on reducing delays around signalised junctions by implementing a system giving priority to public transport vehicles over other traffic. The example chosen for this particular case study is bus line no. 49, a long urban bus line with almost no dedicated bus lanes, which is similar to bus lines that can be found in numerous other cities. The effectiveness of the priority system for decreasing bus delays has already been verified in the planning phase by the city administration through microscopic simulation and the findings have shown that the implementation of the scheme would result in public transport observing 
a decrease of $20-60 \%$ in the number of stops and an increase of 3-6\% in average speed, but also that these improvements would be accompanied by journey time increases for car drivers. This has been deemed reasonable and acceptable, given that the main objective is to improve the efficiency of public transport, and as such, the system has been approved for deployment.

The potential side-effects on pollution and traffic safety, however, have not been part of the administration's initial planning tender, and from the point of view of the stakeholders, a CONDUITS_DST case study towards those effects could bring significant added value. The first stage of this case study (Kaparias et al., 2016) has dealt with the side-effect of pollution, and the analysis has concluded that emission increases of the order of $4-6 \%$ would be expected as a result of the implementation. So in this next stage, it is the safety side-effect that is evaluated.

More specifically, the prospective performance change in terms of accidents, direct safety impacts and indirect safety impacts of the introduction of priority signals along bus line no. 49 is analysed. The study consists of four cases, representing the states before and after the implementation of the system in the morning and evening peak periods, respectively. From the planning phase of the signal control a calibrated PTV VISSIM simulation network has been developed for all four cases (Figure 2). Several simulation runs are carried out over an evaluation period spanning three hours in the respective peak, producing corresponding vehicle record files.

The necessary input data for the three safety KPIs can be directly extracted from the vehicle record files. The evaluation follows a two-stage process, where at first it is conducted at the network level using each of the three KPIs, and then the same procedure is followed for four individual selected network links, so as to demonstrate applicability of the approach in both conditions.
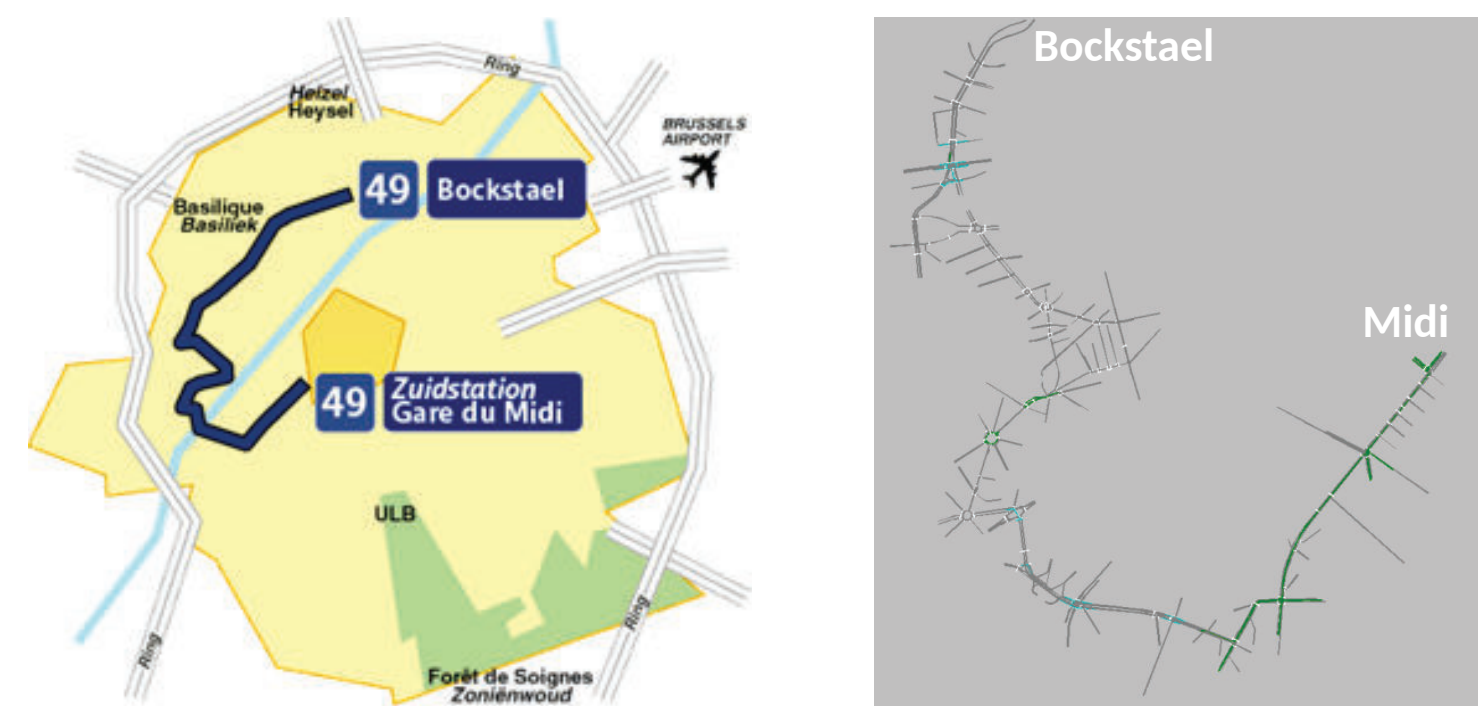

Figure 2: Location of bus line no. 49 in Brussels (left), and simulation network (right)

\subsection{Network-level results}

Using equation (4) the value of $\Delta I_{A C D}$ is calculated as the improvement (positive) or deterioration (negative) of safety performance in terms of accidents. The calculation is carried out separately for the three different accident severity categories (fatality, serious injury, minor injury), which are then aggregated using the weights employed by Kaparias et al. (2012a), i.e. $w_{\text {fat }}=0.85, w_{\text {sinj }}=0.10$ and $w_{\text {minj }}$ $=0.05$ for fatalities, serious and minor injuries, respectively. The calculation is also carried out separately for the different traffic modes (car, HGV, bus, other public transport), so as to get a better mode-specific insight into the impacts.

The results are shown in Table 2, where it can be seen that on average accident reductions of the order of $15 \%$ are foreseen for car and $\mathrm{HGV}$ traffic, arising mostly from the morning peak for cars and from 
the evening peak for HGVs. On the other hand, average increases of the order of $14 \%$ and $23 \%$ are foreseen for the accidents involving buses and other public transport. This is an expected result, as the average speed for car and HGV traffic has decreased after the implementation of the priority scheme, while that of public transport has increased. It should be noted, though, that that these results can be largely attributed to the trends in terms of fatalities, which are most heavily weighted in the KPI calculation, thus reflecting high-level policy objectives (and the near-zero tolerance to fatalities).

\begin{tabular}{|c|c|c|c|c|c|}
\hline \multicolumn{2}{|c|}{$\triangle I_{A C D}$} & Fatality & Serious injury & Minor injury & TOTAL \\
\hline \multirow{3}{*}{ Car } & Morning & 0.245 & 0.190 & 0.131 & 0.234 \\
\hline & Evening & 0.067 & 0.051 & 0.034 & 0.064 \\
\hline & TOTAL & & & & 0.157 \\
\hline \multirow{3}{*}{ HGV } & Morning & 0.055 & 0.042 & 0.028 & 0.052 \\
\hline & Evening & 0.253 & 0.197 & 0.136 & 0.242 \\
\hline & TOTAL & & & & 0.151 \\
\hline \multirow{3}{*}{ Bus } & Morning & -0.041 & -0.028 & -0.061 & -0.119 \\
\hline & Evening & -0.165 & -0.122 & -0.080 & -0.157 \\
\hline & TOTAL & & & & -0.137 \\
\hline \multirow{3}{*}{$\begin{array}{c}\text { Other } \\
\text { public } \\
\text { transport }\end{array}$} & Morning & -0.165 & -0.121 & -0.079 & -0.156 \\
\hline & Evening & -0.320 & -0.231 & -0.149 & -0.302 \\
\hline & TOTAL & & & & -0.226 \\
\hline
\end{tabular}

Table 2: Accidents KPI change between before- and after-evaluation

Making some further considerations about these results, it must be made clear that the estimated increases/decreases are in relation to existing accident numbers, and hence the projected impacts can be deemed significant or not depending on the accident data available. For example, given that accidents involving buses and other public transport vehicles are generally much rarer occurrences than accidents involving cars (usually in the single-digits, mainly because the respective traffic flows are typically considerably lower), the expected safety deterioration for these modes is not as significant as the KPI value suggests; conversely, the projected improvement in terms of accidents involving cars and HGVs is much more significant, as there are usually many more accident occurrences to start with. On the other hand, an effect that cannot be captured by microscopic traffic simulation (and hence by the KPI values) is the potential change in the behaviour of the so-called "active" travellers (pedestrians and cyclists), who may gain confidence due to the slower speeds of car traffic and decide to cross the road more freely. Introducing data from pedestrian simulation modelling in future applications could provide a useful insight into this issue.

\begin{tabular}{|c|c|c|c|c|c|c|c|}
\hline \multirow{2}{*}{$\begin{array}{c}I_{\boldsymbol{D}} \\
\text { (actions/vehicle) }\end{array}$} & \multicolumn{2}{c|}{ Uncontrolled } & \multicolumn{2}{c|}{ Hard/Medium } & \multicolumn{2}{c|}{ Normal } \\
\cline { 2 - 8 } & Before & After & Before & After & Before & After \\
\hline \multirow{4}{*}{ Car } & Morning & 0.020 & 0.023 & 4.77 & 4.89 & 6.75 & 6.62 \\
\cline { 2 - 8 } & Evening & 0.041 & 0.036 & 4.97 & 4.96 & 8.01 & 8.07 \\
\cline { 2 - 8 } & Total & $\mathbf{0 . 0 3 0}$ & $\mathbf{0 . 0 2 9}$ & 4.87 & 4.93 & 7.38 & 7.35 \\
\hline \multirow{3}{*}{ HGV } & Morning & 0 & 0 & 0.98 & 1.07 & 19.09 & 18.78 \\
\cline { 2 - 8 } & Evening & 0 & 0 & 1.40 & 1.41 & 21.15 & 21.25 \\
\cline { 2 - 8 } & Total & $\mathbf{0}$ & $\mathbf{0}$ & $\mathbf{1 . 1 9}$ & $\mathbf{1 . 2 4}$ & $\mathbf{2 0 . 1 2}$ & $\mathbf{2 0 . 0 2}$ \\
\hline \multirow{3}{*}{ Bus } & Morning & 0.071 & 0.075 & 1.34 & 1.57 & 11.14 & 12.33 \\
\cline { 2 - 8 } & Evening & 0.136 & 0.111 & 1.74 & 1.80 & 13.54 & 13.7 \\
\cline { 2 - 8 } & Total & $\mathbf{0 . 1 0 3}$ & $\mathbf{0 . 0 9 3}$ & $\mathbf{1 . 5 4}$ & $\mathbf{1 . 6 8}$ & $\mathbf{1 2 . 3 4}$ & $\mathbf{1 3 . 0 1}$ \\
\hline $\begin{array}{c}\text { Other } \\
\text { public } \\
\text { transport }\end{array}$ & Morning & 0.004 & 0.005 & 1.97 & 0.889 & 9.84 & 6.89 \\
\cline { 2 - 8 } & Evening & 0 & 0 & 1.09 & 1.48 & 6.46 & 4.69 \\
\cline { 2 - 8 } & Total & $\mathbf{0 . 0 0 2}$ & $\mathbf{0 . 0 0 2}$ & $\mathbf{1 . 2 3}$ & $\mathbf{0 . 9 5}$ & $\mathbf{6 . 5 7}$ & $\mathbf{4 . 6 7}$ \\
\hline
\end{tabular}

Table 3: Index for direct safety impacts calculated by braking severity and traffic mode

Considering direct safety impacts, as illustrated by the braking behaviour of drivers around the network, the relevant KPI is calculated using equation (2), and the results, differentiated by braking severity type and traffic mode before and after the implementation of the scheme, are shown in Table 3. As can be seen, the public transport priority system appears to slightly reduce the number of extreme braking 
events per vehicle (with deceleration of more than $7 \mathrm{~m} / \mathrm{s}^{2}$ ), while slightly increasing the number of hard/medium braking occurrences (between $2 \mathrm{~m} / \mathrm{s}^{2}$ and $7 \mathrm{~m} / \mathrm{s}^{2}$ ), especially for buses. This suggests a marginal overall improvement in terms of direct safety impacts. However, an issue potentially requiring additional investigation is the fact that slight increases in the frequency of uncontrolled braking events for all modes are recorded during the morning peak, which are offset by more significant reductions in the evening peak. The reason could be that drivers in the microscopic simulation may be set to drive more hastily in the morning due to being in greater rush to get to work. Further research into user behaviour could confirm whether this effect also occurs in practice and what the implications may be; however, this is beyond the scope of the present study.

As concerns the indirect safety impacts as portrayed by the speeding behaviour of drivers in the network, the relevant KPI is calculated using equation (3), and the results, differentiated by traffic mode, are shown in Table 4. Marginal speeding rate (total time of exceedance of $50 \mathrm{~km} / \mathrm{h}$ over total time of observation in vehicle-hours) reductions can be observed across the board, which can be reflected in the reduced KPI value. Considering the reduction in the total speeding duration over the entire period of observation as a pure number, however, this accounts to almost 40 hours, which is still a significant reduction, even though it may be slightly "downplayed" by the smaller KPI value reduction, which takes into account the traffic volume. As such, it can be concluded that the priority system appears to deliver better safety in terms of indirect impacts. It should be further noted that no speeding occurrences are recorded for public transport, either before or after the implementation of the system. Again, however, it would be interesting in further research to investigate the behavioural dimension of such a finding so as to capture effects, such as how individual drivers respond to the presence of a bus priority system.

\begin{tabular}{|c|c|c|c|c|c|c|c|}
\hline & & \multicolumn{2}{|c|}{ Car } & \multicolumn{2}{|c|}{ HGV } & \multicolumn{2}{|c|}{ Public transport } \\
\hline & & Morning & Evening & Morning & Evening & Morning & Evening \\
\hline \multirow{2}{*}{$\begin{array}{l}\text { Speeding duration } \\
\text { (vehicle-hours) }\end{array}$} & Before & 438.6 & 356.2 & 6.76 & 6.94 & 0 & 0 \\
\hline & After & 407.8 & 347.4 & 6.75 & 7.11 & 0 & 0 \\
\hline \multirow{2}{*}{$\begin{array}{l}\text { Time of observation } \\
\text { (vehicle-hours) }\end{array}$} & Before & 1338 & 1378 & 35.85 & 39.37 & 61.05 & 55.57 \\
\hline & After & 1348 & 1379 & 36.32 & 42.92 & 62.25 & 60.34 \\
\hline \multirow{2}{*}{ Speeding rate } & Before & 0.3275 & 0.2584 & 0.1885 & 0.1762 & 0 & 0 \\
\hline & After & 0.3031 & 0.2519 & 0.1859 & 0.1655 & 0 & 0 \\
\hline \multirow{2}{*}{$I_{I S}$} & Before & \multicolumn{2}{|c|}{0.2925} & \multicolumn{2}{|c|}{0.1821} & \multicolumn{2}{|c|}{0} \\
\hline & After & \multicolumn{2}{|c|}{0.2772} & \multicolumn{2}{|c|}{0.1749} & \multicolumn{2}{|c|}{0} \\
\hline
\end{tabular}

Table 4: Index for indirect safety impacts calculated by traffic mode

\subsection{Link-level results}

Four typical links are selected from abundant links in the network and are evaluated individually as to the safety impacts of the priority system, namely: Link 50, a short straight low trafficked road that includes a roundabout for all modes; Link 93, a long bendy road with high traffic volume for all modes both in the morning and in the evening peak; Link 130, a long straight road with high traffic volume for all modes in the evening peak only; and Link 177, a short straight road with public transport access only. Aside from the topology, the traffic flow characteristics of these links are initially investigated from the simulation data, and it can be found that the average speed has slightly increased on all four of them, and this has often been accompanied with reduced speed variance (and hence more stable traffic flow). The links, therefore, appear to benefit from better mobility following implementation of the priority system, which may, however, be associated with worse safety. In particular in the case of Link 130, the $85^{\text {th }}$ percentile speed exceeds the speed limit, which implies a high speeding rate.

Applying the relative accidents KPI of equation (4) to each of the four links with the same weights as in the network-level evaluation, the results of Table 5 are obtained. As expected given the speed increase, an improvement in terms of accidents is only reported on Link 50 in the morning peak, with decreased index values of different magnitudes found in all other links and times. The highest drop can be identified on Link 130, which can be attributed to the sharp increase in the expected fatality rate, which dominates the calculation due to the higher weight assigned; the severe average speed increase on this 
link explains this result.

\begin{tabular}{|c|c|c|c|c|c|}
\hline \multicolumn{2}{|c|}{$\Delta I_{A C D}$} & Fatality & Serious injury & Minor injury & TOTAL \\
\hline \multirow{3}{*}{ Link 50} & Morning & 0.105 & 0.100 & 0.054 & 0.100 \\
\hline & Evening & -0.223 & -0.211 & -0.106 & -0.211 \\
\hline & TOTAL & & & & -0.035 \\
\hline \multirow{3}{*}{ Link 93} & Morning & -0.207 & -0.151 & -0.098 & 0.052 \\
\hline & Evening & -0.255 & -0.186 & -0.120 & 0.242 \\
\hline & TOTAL & & & & -0.218 \\
\hline \multirow{3}{*}{ Link 130} & Morning & -0.304 & -0.220 & -0.142 & -0.119 \\
\hline & Evening & -0.548 & -0.388 & -0.244 & -0.157 \\
\hline & TOTAL & & & & -0.391 \\
\hline \multirow{3}{*}{ Link 177} & Morning & -0.158 & -0.116 & -0.076 & -0.156 \\
\hline & Evening & -0.106 & -0.078 & -0.051 & -0.302 \\
\hline & TOTAL & & & & -0.125 \\
\hline
\end{tabular}

Table 5: Accidents KPI change between before- and after-evaluation for the four links

Considering direct safety impacts as described by the braking behaviour of drivers, the critical (more than $7 \mathrm{~m} / \mathrm{s}^{2}$ ) occurrences and the corresponding KPI values calculated through equation (2) for each of the four links are given in Table 6 . The results suggest that the implemented priority system generates positive direct safety impacts on the links with lower average traffic volume (50 and 130). However, a negative direct safety impact appears to arise on the more heavily trafficked Link 93, attributed mostly to the morning peak. As concerns the very lightly trafficked Link 177, this cannot be considered as statistically significant, as only a single occurrence of critical braking is identified.

\begin{tabular}{|c|c|c|c|c|c|}
\hline \multicolumn{2}{|c|}{} & Link 50 & Link 93 & Link 130 & Link 177 \\
\hline \multirow{2}{*}{ Morning } & Before & 2 & 7 & 3 & 0 \\
\cline { 2 - 6 } & After & 1 & 9 & 2 & 0 \\
\hline \multirow{2}{*}{ Evening } & Before & 3 & 9 & 5 & 1 \\
\cline { 2 - 6 } & After & 2 & 5 & 4 & 0 \\
\hline \multirow{2}{*}{ TOTAL } & Before & $\mathbf{5}$ & $\mathbf{1 6}$ & $\mathbf{8}$ & $\mathbf{1}$ \\
\cline { 2 - 6 } & After & $\mathbf{3}$ & $\mathbf{1 4}$ & $\mathbf{6}$ & $\mathbf{0}$ \\
\hline \multirow{2}{*}{$I_{\boldsymbol{D S}}$ (actions / 1000 vehicles) } & Before & 3.3 & 3.7 & 5.6 & 10.6 \\
\cline { 2 - 6 } & After & 2.7 & 4.1 & 1.6 & 0 \\
\hline
\end{tabular}

Table 6: Critical braking occurrences and direct safety impacts KPI values for the four links

With respect to the indirect safety impacts evaluation on the basis of the speeding behaviour of the drivers, the corresponding KPI values calculated through equation (3) for each of the four links are given in Table 7. It can be seen that on the links with lower speeding rate (Links 50 and 93), this appears to be very marginally (almost negligibly) increased after the implementation of the priority system. On the other hand, on the link with higher speeding rate (130), a drop is reported. No speeding is reported on Link 177, which is expected, as this is a public transport access only link. As such, the results suggest that the priority system appears to have a positive indirect safety impact on links with high speeding.

\begin{tabular}{|c|c|c|c|c|c|c|c|c|c|}
\hline \multirow{2}{*}{\multicolumn{2}{|c|}{ Speeding rate }} & \multicolumn{2}{|c|}{ Link 50} & \multicolumn{2}{|c|}{ Link 93} & \multicolumn{2}{|c|}{ Link 130} & \multicolumn{2}{|c|}{ Link 177} \\
\hline & & Morning & Evening & Morning & Evening & Morning & Evening & Morning & Evening \\
\hline \multirow{4}{*}{$I_{I S}$} & Before & 0.0131 & 0.0168 & 0.0546 & 0.0511 & 0.2513 & 0.1868 & $\mathbf{0}$ & $\mathbf{0}$ \\
\hline & After & 0.0147 & 0.0151 & 0.0560 & 0.0547 & 0.1312 & $\mathbf{0 . 2 3 2 7}$ & $\mathbf{0}$ & $\mathbf{0}$ \\
\hline & Before & \multicolumn{2}{|c|}{0.0145} & \multicolumn{2}{|c|}{0.0529} & \multicolumn{2}{|c|}{0.2212} & \multicolumn{2}{|c|}{$\mathbf{0}$} \\
\hline & After & \multicolumn{2}{|c|}{0.0149} & \multicolumn{2}{|c|}{0.0552} & \multicolumn{2}{|c|}{0.1837} & \multicolumn{2}{|c|}{ 0 } \\
\hline
\end{tabular}

Table 7: Indirect safety impacts KPI values for the four links

\subsection{Results in a broader policy-related context}

Summarising the results of the evaluation, the public transport priority system is expected to have a generally positive safety effect at the network- and the link-levels in terms of accidents, direct safety 
impacts and indirect safety impacts. However, some areas of potentially adverse safety impacts can be identified. At the network-level these include slight increases in accidents involving buses and public transport vehicles, and marginally higher frequency of uncontrolled braking occurrences during the morning peak. At the level of the individual links analysed, they are mostly related to increases in accident risk and in direct safety impacts for the more heavily-trafficked links.

Clearly, some of the negative results obtained could be seen as a case against the implementation of the scheme, despite the great, and possibly even more significant, benefits that may be delivered with respect to other aspects (e.g. in terms of mobility efficiency). As such, these results should not be viewed in isolation, but rather as an integral part of a bigger and broader analysis, whereby the identified potential negative impacts can be identified so that efforts can focus on devising ways to mitigate them.

For instance, in the case of Brussels, previous work by the authors (Kaparias et al., 2016) has found that, despite great benefits in terms of the mobility efficiency of public transport passengers, the immediate introduction of the public transport priority system will inevitably have a negative short-term effect on pollution, mainly due to the reduction of the green time for car traffic. This will be likely gradually mitigated by changes in route and mode choice, but as is commonly the case with the procurement procedures of such schemes, resources to estimate such effects that are beyond the direct goal of the scheme (i.e. the improvement of the mobility of public transport passengers) are generally not provided.

Using CONDUITS_DST, it has been possible to not only quantify this short-term negative side-effect, but also to estimate the approximate change in traffic volumes that is necessary to mitigate it; indeed, sensitivity analysis of the corresponding pollution KPI values has shown that a $4 \%$ decrease in the relevant traffic volumes during the evening peak would completely eliminate the negative pollution side-effect of the priority scheme (Kaparias et al., 2012b). As a result, the public administration of Brussels has been able to use this result to evaluate whether this decrease can be achieved with other traffic management measures that are already under implementation, such parking management, or whether additional new measures need to be planned. A similar sensitivity analysis could be conducted with respect to the identified safety impacts, but this will be reported in future work by the authors.

\section{CONCLUSIONS}

A predictive evaluation framework for the theme of traffic safety of road traffic management has been introduced in this paper, with the objective of being used in conjunction with the CONDUITS KPIs and being implemented as a dedicated module to the CONDUITS_DST software. A number of models and metrics linking traffic characteristics with road safety impacts have been identified and proposed, which enable the extraction of the necessary input data for each of the three CONDUITS KPIs for traffic safety (accidents, direct impacts, and indirect impacts) directly from microscopic traffic simulation models. The proposed models and metrics have then been tested in conjunction with the KPIs using data from simulation models before and after the implementation of a bus priority signalling system in the city of Brussels. Testing has been carried out both at the network- and at the individual-link-level, and the results appear to capture the expected safety impacts adequately well.

Naturally, a limitation of the proposed work is that it has not been comprehensively validated. Unfortunately, validation would necessitate real-world safety impact data (typically accident records) so as to enable comparisons with the predicted impacts, but such data usually take several months or even years to accumulate, and even then they are often not reliable enough to offer statistically significant conclusions (e.g. even after several years, fatal accident occurrences are often in the singledigit numbers). Consequently, the proposed approach is not intended to offer accurate predictions of actual accident and/or casualty numbers. What it can offer, however, is a priori estimates of how safety levels as a whole may change as a result of the implementation of a scheme, which can then inform policy-making before anything is deployed on the ground. From the point of view of practitioners this is a very valuable insight, as it allows them to perform a multi-perspective evaluation that includes multiple and diverse fields of policy, which may lie beyond their direct remit. It also gives them the 
means to communicate results to political stakeholders (who often have a non-technical background) and to assess side-scenarios on a higher level of abstraction. In the concrete case of Brussels, the present study has made a valuable contribution to the city's pursuit of high level evaluation means for the verification of measures relating to Sustainable Urban Mobility Plans.

While the present study has shed some light on the topic of predictive evaluation of traffic safety impacts, work in this direction continues. It is an essential next step for the proposed models and metrics to be incorporated into the CONDUITS_DST software, complementing the already integrated and validated pollution and traffic efficiency modules. Apart from the user-friendliness of the evaluation process, the integration will additionally enable the consideration of traffic safety as part of a broader multi-objective problem alongside the other themes, rather than individually. This means that solutions will be able to be compared by decision-makers directly on the basis of their KPI values in the different themes, thus providing a more informed and comprehensive evaluation process. As it is likely that tradeoffs will have to be made between meeting objectives in different themes to varying extents, the decision-making process can become a highly complex problem, opening great prospects of further research in the development of multi-objective optimisation algorithms using the different KPIs as decision variables to facilitate this.

\section{ACKNOWLEDGEMENTS}

The authors would like to thank Kapsch TrafficCom for sponsoring the work reported here as part of the CONDUITS-DST project, and the Brussels-Capital Region for providing the data and microscopic traffic simulation models used.

\section{REFERENCES}

Bared, J., Giering, G.L., Warren, D.L., 1999. Safety evaluation of acceleration and deceleration lane lengths. ITE Journal. 69, 50-54.

Bowie, N.N., Waltz, M., 1994. Data-analysis of the speed related crash issue. Auto and Traffic Safety. 2, 31-38.

Cambridge Systematics Inc., PB Consult Inc., System Metrics Group, 2005. Analytical tools for asset management. NCHRP report 545, Transportation Research Board.

Cianfano, M., Gentile, G., Nussio, F., Tuffanelli, G., Rossi, P, Vergini, R., Terenzi, G., 2011. Transport and mobility indicators based on traffic measures - Good practices guidebook. CITEAIR II Deliverable.

Eden, N., Tsakarestos, A., Kaparias, I., Gal-Tzur, A., Schmitz, P., Hauptmann, S., Hoadley, S., 2012. Using Key Performance Indicators for traffic management and Intelligent Transport Systems as a prediction tool. $19^{\text {th }}$ World Congress on Intelligent Transport Systems, Vienna, Austria.

Eden, N., Kaparias, I., Tsakarestos, A., Gal-Tzur, A., Schmitz, P., Hoadley, S., Harrich, M., 2013. Using Key Performance Indicators for multi-criteria traffic management strategic decisions. $9^{\text {th }}$ European Intelligent Transport Systems Congress, Dublin, Ireland.

Elvik, R., Høye, A., Vaa, T., Sørensen, M., 2009. The handbook of road safety measures. Bingley, Emerald Group Publishing Ltd.

European Commission, 2001. European transport policy for 2010: time to decide. White Paper.

European Commission, 2011. Roadmap to a single European Transport Area - Towards a competitive and resource efficient transport system. White Paper.

European Transport Safety Council, 2001. Transport Safety Performance Indicators.

Handel, P. Skog, I., Wahlstrom, J., Bonawide, F., Welsh, R., Ohlsson, J., Ohlsson, M., 2014. Insurance telematics: Opportunities and challenges with the smartphone solution. IEEE Intelligent Transportation Systems Magazine. 6, 57-70.

Hydén, C., 1987. The development of a method for traffic safety evaluation: The Swedish traffic conflicts technique. Department of Technology and Society, Lund University. 
Hydén, C., 1996. Traffic conflicts technique: State-of-the-art, in: Topp, H.H. (Ed.), Traffic safety work with video processing, University Kaiserslautern, Transportation Department, Green Series, 37, pp. $3-14$.

Kaparias, I., Bell, M.G.H., Eden, N., Gal-Tzur, A., Komar, O., Prato, C.G., Tartakovsky, L., Aronov, B., Zvirin, Y., Gerstenberger, M., Tsakarestos, A., Nocera, S., Busch, F., 2011. Key Performance Indicators for traffic management and Intelligent Transport Systems. CONDUITS Deliverable 3.5.

Kaparias, I., Eden, N., Tsakarestos, A., Gal-Tzur, A., Gerstenberger, M., Hoadley, S., Lefebvre, P., Ledoux, J., Bell, M.G.H., 2012a. Development and application of an evaluation framework for urban traffic management and Intelligent Transport Systems. Transport Research Arena Europe 2012, Athens, Greece.

Kaparias, I. Eden, N., Tsakarestos, A., Zarkhin, N., Böhm, C., 2012b. Development and application of the CONDUITS Decision Support Tool for predictive assessment of pollution impacts. 1st Annual Report of the CONDUITS-DST project.

Kaparias, I., Manassa, E., Eden, N., Tsakarestos, A., Schmitz, P, Hoadley, S., Hauptmann, S., 2016. Policy-aware assessment of environmental impacts from transport in smart cities. Journal of Smart Cities. 2, 1-9.

Nilsson, G., 2004. Traffic safety dimensions and the power model to describe the effect of speed on safety. Lund Institute of Technology and Society, Traffic Engineering. Bulletin 221.

Organisation for Economic Cooperation and Development (OECD), 2006. Speed management. European Conference of Ministers of Transport, Paris, France.

Sigfridsson, S.E., Heijster, C., Lang, J., Plaza Rubio, J.A., Stoop, J., Taylor, F., Valkama-Joutsen, P., Walta, W., 2001. Transport accident and incident investigation in the European Union. European Transport Safety Council report.

STIB-MIVB, 2019. Official website. www.stib-mivb.be.

Tsakarestos, A., Nocera, S., Kaparias, I., Eden, N., 2011. Performance evaluation of urban traffic management and ITS: The Rome case study. 2011 SIDT National Conference and Scientific Seminar, Venice, Italy.

US National Association of Insurance Commissioners (NAIC), 2019. Usage-based insurance and telematics. https://www.naic.org/cipr topics/topic usage based insurance.htm

Zavitsas, K., Kaparias, I., Bell, M.G.H., 2011. Transport problems in cities. CONDUITS Deliverable 1.1 .

Zhao, Y., 2002. Telematics: Safe and fun driving. IEEE Intelligent Systems. 17, 10-14.

Zhou, P. Ang, B.W., Poh, K.L., 2006. Comparing aggregating methods for constructing the composite environmental index: An objective measure. Ecological Economics. 59, 305-311. 